\title{
RESEARCH
}

\section{RELATIONSHIP BETWEEN THE PROGNOSTIC NUTRITIONAL INDEX AND ALL-CAUSE MORTALITY IN ELDERLY PATIENTS WITH NON-ST SEGMENT ELEVATION MYOCARDIAL INFARCTION}

Turkish Journal of Geriatrics

DOI: $10.31086 /$ tigeri.2021.234

$2021 ; 24(3): 370-380$

- Özge Çakmak KARAASLAN ${ }^{1}$

- Cem ÇÖTEL ${ }^{1}$

- Hasan Can KÖNTE 2

- Murat Oğuz ÖZiLHAN ${ }^{1}$

- Orhan MADEN ${ }^{1}$.

\section{Abstract}

Background: Patients over 65 years of age with non-ST segment elevation myocardial infarction are at higher risk of mortality and morbidity than younger patients. The prognostic nutritional index is a combined immunologicalnutritional status score based on serum albumin levels and lymphocyte values. We evaluated the association between prognostic nutritional index value and all-cause mortality in elderly patients with non-ST segment elevation myocardial infarction. The current study presents the first evaluation of prognostic nutritional index in elderly patients with non-ST segment elevation myocardial infarction.

Methods: This was a retrospective observational study. The study population was divided into two groups according to their admission prognostic nutritional index. A prognostic nutritional index of 46 was determined as the optimal cutoff value to predict the primary endpoint, which was all-cause death during the follow-up period.

Results: Compared to patients with a prognostic nutritional index $\geq 46$, those with a prognostic nutritional index $<46$ were older $(p<0.001)$ and more often had a history of hypertension and known coronary artery disease $(p=0.001)$. All-cause mortality was significantly higher in the group with lower prognostic nutritional index (12.5\% vs $4.8 \% ; p=0.007)$. The prognostic nutritional index predicted the primary endpoint, and this prediction was statistically significant (sensitivity $71 \%$; specificity $67 \%$ ). Univariate Cox regression analyses and multivariate Cox regression analyses showed that a cut-off level of prognostic nutritional index $<46$ was significantly associated with the primary endpoint.

Conclusions: The prognostic nutritional index score was associated with

${ }^{1}$ Ankara City Hospital, Department of Cardiology, Ankara, Turkey

2 Kahramankazan State Hospital, Department of Cardiology, Ankara, Turkey

Keywords: Mortality; Non-ST Elevated Myocardial Infarction; Nutrition Assessment. 


\section{INTRODUCTION}

The numbers and proportion of the population aged over 65 years are increasing due to extended lifespans, health improvements and decreasing birth rates (1). Of those who die from ischaemic heart disease, $83 \%$ are over the age of 65 years. Elderly patients with non-ST-segment elevation myocardial infarction (NSTEMI) are at higher risk of mortality and morbidity than younger patients (2). The current guidelines recommend evaluating invasive treatments and considering personal characteristics, such as estimated life expectancy, comorbidities, quality of life and patient preference, after a careful risk/benefit evaluation in elderly patients with NSTEMI (3). Therefore, clinical scores for risk assessment are of great importance in choosing the appropriate treatment and evaluating mortality and morbidity risk in elderly patients with NSTEMI.

Malnutrition is an important risk factor for cardiovascular disease (CVD) in the elderly population and has a known association with a prolonged hospital stay, increased mortality and reduced quality of life (4-6). Malnutrition has been shown to associate with poor prognosis and mortality in cardiovascular disease, such as ST-segment elevation myocardial infarction (STEMI) and heart failure $(7,8)$. Therefore, routine screening of the elderly population in terms of nutrition has been suggested (9). Nevertheless, evaluating nutritional status is not simple, as different components of malnutrition need to be considered. The Prognostic Nutritional Index (PNI) reflects a combined score of an immunological nutritional status based on serum albumin levels and lymphocyte values (10). This index is easy to calculate, as it requires only simple blood parameters and is a cost-effective method. Recent studies have shown that nutritional status evaluated by PNI is an independent prognostic factor in patients with different CVD $(7,8)$. However, the association of PNI with mortality has not been reported in elderly patients with NSTEMI. Therefore, in this study, we evaluated the association between the value of $\mathrm{PNI}$ and all-cause mortality in elderly patients with NSTEMI. The current study presents the first evaluation of PNI in elderly patients with NSTEMI.

\section{METHODS}

Between March 2019 and August 2020, 404 consecutively confirmed patients aged $\geq 65$ with NSTEMI who were admitted to the emergency department of our institution were retrospectively evaluated. NSTEMI was determined by the appropriate guidelines (11). Patients with an active infection, a history of chronic inflammatory disease, a known neoplasm, severe hepatic disease, an advanced-stage renal disorder or missing clinical data, as well as individuals undergoing renal replacement therapy, were excluded from this study.

The PNI was calculated based on the serum albumin values and the total lymphocyte count obtained at admission: $10 \times$ albumin $(\mathrm{g} / \mathrm{dl})+0.005 \times$ total lymphocyte count (permm3). The PNI was calculated for each participant. The study population ( $n=376)$ was divided into two groups according to their admission PNI. The cut-off value of PNI to predict the primary endpoint was evaluated by receiver operating characteristic (ROC) curve analysis. A PNI of 46 was determined as an optimal cut-off value to predict the primary endpoint. The study population groups were organised accordingly: $\mathrm{PNI}<46(n=$ $128)$ and $P N I \geq 46(n=248)$.

The study protocol was reviewed and approved by the University of Health Sciences, Ankara City Hospital Ethics Committee in accordance with the Declaration of Helsinki (approval no; E1/21/1487, date; 03 February 2021).

NSTEMI was defined as the presence of cardiac biomarkers, such as cardiomyocyte necrosis and increased troponin $\mathrm{T}$ ( $\mathrm{cTnT}$ ) with clinically concordant myocardial ischaemia and without ST segment elevation. The primary endpoint was all-cause death during the follow-up period. All-cause mortality was defined as death from any cause during hospitaliza- 
tion or after discharge during the follow-up period. The mortality data of the participants were obtained by the medical records, while the details and the causes of death were obtained from the National Survival Registry. Cardiovascular death was defined as death due to myocardial infarction, death due to heart failure, death due to malignant arrhythmia or sudden cardiac arrest because of other or unknown causes during hospitalization and after discharge.

The baseline clinical and demographic characteristics of the patients were gathered from the hospital's medical database. Left ventricular ejection fraction (LVEF) was calculated using the modified Simpson method within 24 hours of admission (12). An electrocardiogram (ECG) was examined on admission in the emergency unit, after the procedure and daily during the hospital stay. Venous blood samples from the antecubital vein were obtained using standardized EDTA blood tubes within 10-30 minutes of admission to the hospital. A complete blood count including the white blood cell (WBC), haemoglobin and lymphocyte levels was measured using an automated haematology analyzer system (Beckman Coulter Ireland, Inc, Galway, Ireland). Albumin levels were measured using the photometric method (DC 800; Beckman Coulter, Dublin, Ireland).

According to the ESC guidelines, an immediate invasive strategy was performed on patients with at least one very-high-risk NSTEMI criteria (11); all the remaining patients underwent coronary angiography (CAG) within 48 hours after admission with a diagnosis of NSTEMI. The heart team determined the appropriate revascularization strategy (percutaneous coronary intervention or coronary artery bypass grafting) after CAG. For the patients whose coronary artery anatomy was not suitable for intervention treatment, pharmacological treatment was arranged. All the patients in the study received a loading dose of aspirin and a loading dose of P2Y12 receptor inhibitors on admission. The patients received 70-100 U/kg of intravenous unfractionated heparin before the procedure. The use of a glyco- protein Ilb/IIla inhibitor (tirofiban) was at the discretion of the primary operator. Most of the percutaneous coronary intervention $(\mathrm{PCl})$ procedures were performed using a transradial approach; a transfemoral approach was used on the patients who were not suitable for the transradial approach. The appropriate P2Y12 inhibitor therapy and $81-100 \mathrm{mg}$ aspirin per day were prescribed during the hospitalization and at discharge according to the current guidelines (11).

All the data were analysed using the SPSS 22.0 Statistical Package Program for Windows (SPSS; IBM, Armonk, New York, USA). A KolmogorovSmirnov test was utilized for the assessment of the normality of distribution. Continuous variables were presented as mean \pm standard deviation and median with interquartile ranges and categorical variables as the number of patients and percentages. A comparison between groups was made with a Student's t-test for normally distributed variables and a Mann-Whitney $U$ test for variables without normal distribution. Categorical data from both groups were compared using the $\mathbf{X}^{2}$ or Fisher's exact test. Univariate and multivariate analyses with Cox proportional hazards regression were used to evaluate the association between PNI and all-cause mortality. Variables displaying $\mathrm{p}<0.05$ in the univariable analysis were used in a multivariable logistic regression analysis. Survival estimates were calculated by the Kaplan-Meier method, and the log-rank tests were used for comparison. The ability of the PNI value to predict the primary endpoint was evaluated by ROC curve analysis and area under curve (AUC) values. The cut-off value was calculated according to the Youden index. A p-value $<0.05$ (using a two-sided test) was accepted as significant.

\section{RESULT}

Initially, a total of 404 consecutive NSTEMI patients aged $\geq 65$ were considered for the study; 28 patients were excluded because of having at least one of the exclusion criteria. Consequently, 376 
patients were enrolled in the current study. In the study population, the mean age was $73 \pm 7.1$ years, the male gender ratio was $60 \%$, and the mean follow-up time was $335 \pm 117$ days. The cut-off value of PNI for predicting the primary endpoint was 46 (sensitivity $71 \%$, specificity $=67 \%$ ) according to the Youden index. We divided the patients into two groups according to the PNI cut-off value; 128 patients had a PNI score < 46, and 248 patients had a $\mathrm{PNI} \geq 46$. Demographic characteristics and clinical and laboratory values of the study population are presented in Table 1. Compared to the patients with $\mathrm{PNI}$ scores $\geq 46$, those with PNI scores $<46$ had an advanced age $(p<0.001)$ and had a history of hypertension (HT) $(p=0.008)$ and known coronary artery disease (CAD) ( $p=0.001)$ more often. However, the histories of the patients were similar in terms of diabetes mellitus (DM), hyperlipidemia $(\mathrm{HPL})$, previous heart failure and stroke. The patients with a PNI score $<46$ had significantly lower blood albumin ( $p<0.001)$, lower total cholesterol $(p=0.010)$, a lower glomerular filtration rate $(p<$ $0.001)$, lower WBC counts $(p=0.012)$, lower haemoglobin levels $(p<0.001)$, lower lymphocyte counts $(p<0.001)$ and higher $C$-reactive protein (CRP) levels $(p=0.011)$ compared with those with a PNI score $\geq 46$. No significant differences were noted in systolic blood pressure (SBP), heart rate and LVEF at admission between the two groups. The patients in both groups had similar treatments with respect to aspirin, PY212 receptor inhibitors, B-blockers, statins and angiotensin-converting enzyme (ACE) inhibitors or angiotensin II receptor blockers. The ratio of diuretic therapy prescribed at discharge was higher in the low-PNI group ( $p=0.009$ ).

The ratios of patients who underwent coronary artery bypass grafting as a revascularization strategy, who underwent $\mathrm{PCl}$, and who were followed up medically without invasive intervention were similar in both groups (Table 1).

Table 1. Baseline clinical, echocardiographic, and laboratory characteristics of patients stratified by values of the prognostic nutritional index.

\begin{tabular}{|c|c|c|c|}
\hline & \multicolumn{2}{|c|}{ Prognostic Nutritional İndex } & \multirow{2}{*}{$p$ value } \\
\hline & $<46(n=128)$ & $\geq 46(n=248)$ & \\
\hline Age (year) & $78 \pm 12.7$ & $70 \pm 7.0$ & $<0.001$ \\
\hline Male, n (\%) & $68(53.1)$ & $160(64.5)$ & 0.032 \\
\hline Heart Rate, BPM & $78 \pm 11.0$ & $78 \pm 21.0$ & 0.990 \\
\hline $\mathrm{SBP}, \mathrm{mmHg}$ & $120 \pm 27.5$ & $130 \pm 16.3$ & 0.062 \\
\hline LVEF, \% & $45 \pm 10.0$ & $45 \pm 15.0$ & 0.497 \\
\hline \multicolumn{4}{|l|}{ History, n (\%) } \\
\hline Diabetes mellitus & $76(59.3)$ & $128(51.6)$ & 0.152 \\
\hline Hypertension & $96(75)$ & $152(61.2)$ & 0.008 \\
\hline Hyperlipidemia & $20(16.6)$ & $48(19.3)$ & 0.373 \\
\hline History of heart failure & $48(37.5)$ & $96(38.7)$ & 0.332 \\
\hline Known coronary artery disease & $44(34.3)$ & $120(48.3)$ & 0.001 \\
\hline History of Stroke & $16(12.5)$ & $16(6.4)$ & 0.053 \\
\hline
\end{tabular}




\begin{tabular}{|c|c|c|c|}
\hline \multicolumn{4}{|l|}{ Admission Laboratory Variables } \\
\hline Creatinine (mg/dl) & $0.8 \pm 0.3$ & $0.8 \pm 0.3$ & 0.180 \\
\hline Albumin (g/dl) & $37.0 \pm 2.0$ & $41.4 \pm 4$ & $<0.001$ \\
\hline Total cholesterol (mg/dL) & $165 \pm 50$ & $171 \pm 62$ & 0.010 \\
\hline $\mathrm{LDL}(\mathrm{mg} / \mathrm{dL})$ & $104 \pm 39$ & $104 \pm 45$ & 0.297 \\
\hline eGFR (ml/min/1.73m²) & $74 \pm 31$ & $87 \pm 29$ & $<0.001$ \\
\hline WBC $\left(x 10^{3} / \mu \mathrm{L}\right)$ & $8.6 \pm 3.0$ & $9.35 \pm 3.44$ & 0.012 \\
\hline Hemoglobin (mg/dL) & $13.1 \pm 2.1$ & $13.5 \pm 2.1$ & $<0.001$ \\
\hline Lymphocyte $\left(\times 10^{3} / \mu \mathrm{l}\right)$ & $0.97 \pm 0.39$ & $1.90 \pm 0.64$ & $<0.001$ \\
\hline CRP & $8.23 \pm 39.89$ & $5.71 \pm 6.90$ & 0.011 \\
\hline Troponin T (ng/mL) & $3.222 \pm 9.672$ & $2.433 \pm 8.165$ & 0.671 \\
\hline \multicolumn{4}{|l|}{ Coronary Artery Disease, n (\%) } \\
\hline Left Main & $8(6.2)$ & 0 & 0.002 \\
\hline Left Anterior Descending & $56(43.7)$ & $120(48.3)$ & 0.913 \\
\hline Left Circumflex & $52(40.6)$ & $116(46.7)$ & 0.258 \\
\hline Right Coronary Artery & $28(21.8)$ & $120(48.3)$ & 0.230 \\
\hline \multicolumn{4}{|l|}{ Angiographic Data, n (\%) } \\
\hline Syntax Score & $10 \pm 8.7$ & $9 \pm 9.7$ & 0.674 \\
\hline TIMI Thrombus Grade & $4.0 \pm 2.0$ & $3.0 \pm 1.8$ & 0.683 \\
\hline \multicolumn{4}{|l|}{ Selection Of Revascularization, n (\%) } \\
\hline Coronary Artery Bypass Grafting & $8(6)$ & $32(12.5)$ & 0.128 \\
\hline Percutaneous Coronary Intervention & $100(78.1)$ & 188(75.8) & 0.615 \\
\hline Non-Interventional Treatment & $20(15)$ & $32(12)$ & 0.469 \\
\hline \multicolumn{4}{|l|}{ Medications At Discharge, n (\%) } \\
\hline Aspirin & $127(99.2)$ & $244(98.4)$ & 0.505 \\
\hline B-blocker & $116(90.6)$ & $228(91.9)$ & 0.328 \\
\hline Statins & $120(93.8)$ & $236(95.2)$ & 0.180 \\
\hline ACEls or ARBs & $120(93.8)$ & $232(93.5)$ & 0.588 \\
\hline Diuretics & $36(28.1)$ & $60(24.2)$ & 0.009 \\
\hline P2Y12 receptor inhibitors & $125(97.7)$ & $242(97.6)$ & 0.964 \\
\hline
\end{tabular}

SBP: Systolic Blood Pressure; LVEF: Left ventricular ejection fraction; LDL: Low-density lipoprotein cholesterol; eGFR: estimated glomerular filtration rate; WBC: White blood cell; CRP: C-reactive protein; Syntax: SYNergy between PCI with TAXUS and Cardiac Surgery; TIMI: Thrombolysis in Myocardial Infarction; ACEI: Angiotensin-converting enzyme inhibitör; ARB: Angiotensin II receptor blockers 
Table 2 presents the primary endpoint of the study according to the PNI values. During the follow-up period, all-cause death occurred in 28 of 376 subjects (7.4\%). All-cause mortality was significantly higher in the group with lower PNI scores (12.5\% vs. $4.8 \%$; $P=0.007)$. Although the incidence of cardiovascular death was higher in the low-PNI group, it was not statistically significant.

The ability of the PNI to predict the primary endpoint was evaluated by ROC curve analysis. The AUC value of this analysis is presented in Figure 1 ( $A \cup C=0.636,95 \% \mathrm{Cl}=0.502-0.771, \mathrm{p}=0.016) . \mathrm{It}$ was determined that the PNI predicted the primary endpoint, and this prediction was statistically significant (Sensitivity $71 \%$, specificity $=67 \%$ ).

The patients were followed up for a mean period of $335 \pm 117$ days. The Kaplan-Meier analysis showed a significantly lower primary endpoint-free survival rate in patients with low PNI scores (logrank, $P=0.008$ ) (Figure 2).

Univariate Cox regression analyses showed that the SYNTAX score, known CAD, admission heart

Figure 1. Receiver-operating characteristic curve of the $\mathrm{PNI}$ for predicting primary endpoint.

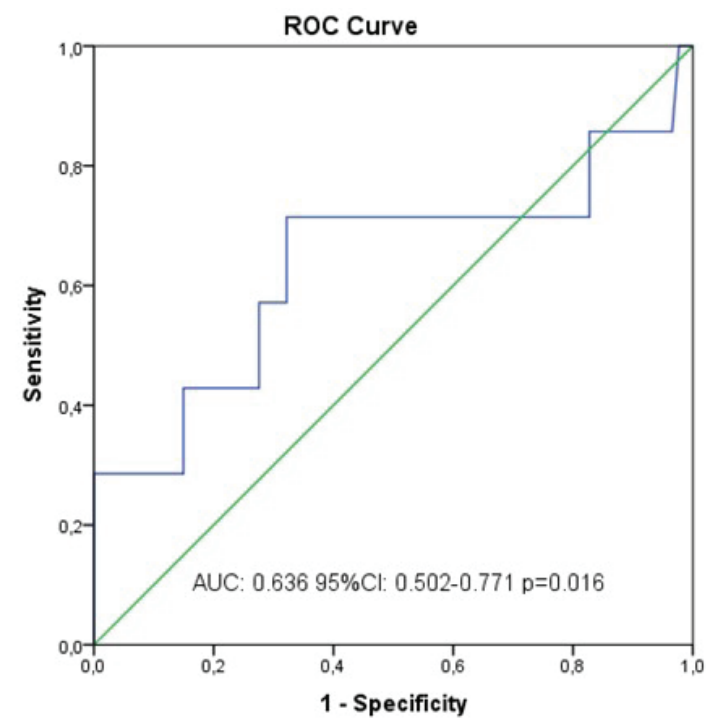

rate, SBP, WBC and a cut-off level of PNI $<46$ was significantly associated with the primary endpoint, as shown in Table 3. All-cause mortality had higher rates at lower PNI levels (hazard ratio: 0.328; 95\% confidence interval 0.123-0.872; $p=0.025)$. Other multivariate independent predictors of mortality were admission heart rate (hazard ratio, 1.060; 95\% confidence interval, 1.029-1.093], $P<0.001$ ), LVEF (hazard ratio, 0.938; 95\% confidence interval, $0.887-0.991, P=0.022$ ) and WBC count (hazard ratio, 1.212; $95 \%$ confidence interval, 1.105-1.329, $\mathrm{P}<$ 0.001 ) (Table 3).

\section{DISCUSSION}

To the best of our knowledge, this is the first study to investigate the prognostic value of $\mathrm{PNI}$ in patients aged $\geq 65$ with NSTEMI. The major findings were as follows: the low PNI group had a higher all-cause mortality rate compared to the high-PNI group, even though both groups had similar medical histories, such as DM, heart failure, previous stroke and HPL. HT and known CAD were more common in

Figure 2. Kaplan-Meier curve analysis of the composite primary endpoint.

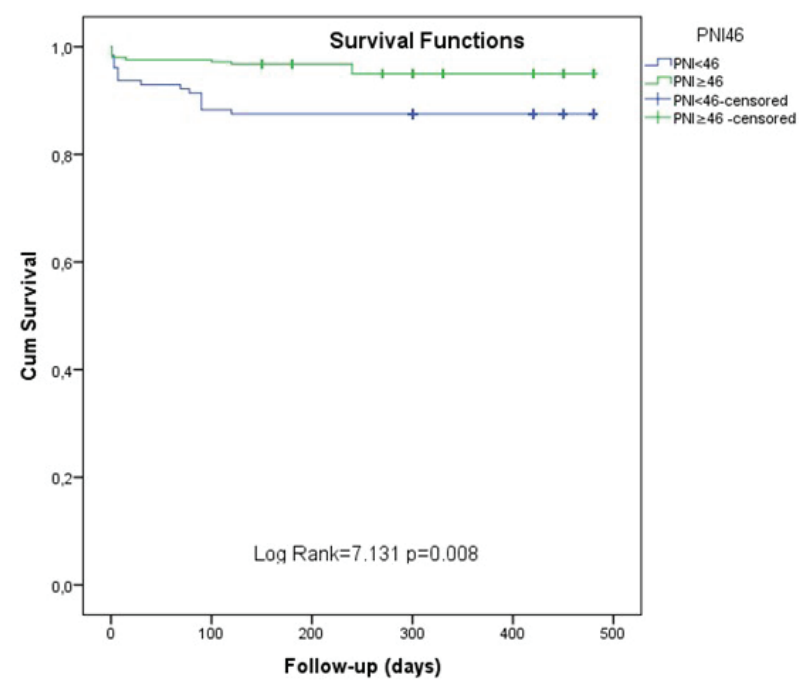


Table 2. The Primary Endpoint of patients classified by prognostic nutritional index

\begin{tabular}{|l|c|c|c|}
\hline & PNI<46 $(\mathbf{n = 1 2 8})$ & PNI $\mathbf{4 6}(\mathbf{n = 2 4 8})$ & P value \\
\hline All cause mortality & $16(12.5)$ & $12(4.8)$ & 0.007 \\
\hline Cardiovascular death & $8(6.2)$ & $12(4.8)$ & 0.563 \\
\hline Follow-up time, day & $300 \pm 150$ & $315 \pm 150$ & 0.069 \\
\hline
\end{tabular}

PNI: Prognostic Nutritional Index

Table 3. Univariate and Multivariate cox proportional hazard models for prediction of primary endpoint.

\begin{tabular}{|c|c|c|c|c|}
\hline & $\mathrm{HR}(95 \% \mathrm{CI})$ & $p$ value & $\mathrm{HR}(95 \% \mathrm{Cl})$ & $p$ value \\
\hline SYNTAX Score & $1.073(1.037-1.111)$ & $<0.001$ & $1.046(0.988-1.107)$ & 0.121 \\
\hline Known coronary artery disease & $0.303(0.133-0.687)$ & 0.004 & $0.902(0.517-1.573)$ & 0.717 \\
\hline Heart Rate, BPM & $1.087(1.062-1.113)$ & $<0.001$ & $1.060(1.029-1.093)$ & $<0.001$ \\
\hline $\mathrm{SBP}, \mathrm{mmHg}$ & $0.928(0.909-0.947)$ & $<0.001$ & $1.004(0.974-1.035)$ & 0.802 \\
\hline LVEF & $0.916(0.884-0.950)$ & $<0.001$ & $0.938(0.887-0.991)$ & 0.022 \\
\hline Creatinine (mg/dl) & $3.030(0.918-10.005)$ & 0.069 & & \\
\hline eGFR & $0.987(0.969-1.006)$ & 0.176 & & \\
\hline Total cholesterol (mg/dL) & $1.000(0.992-1.009)$ & 0.923 & & \\
\hline $\mathrm{LDL}(\mathrm{mg} / \mathrm{dL})$ & $1.002(0.992-1.013)$ & 0.681 & & \\
\hline WBC (x103/ML) & $1.214(1.155-1.276)$ & $<0.001$ & $1.212(1.105-1.329)$ & $<0.001$ \\
\hline Hemoglobin (mg/dL) & $1.053(0.868-1.277)$ & 0.601 & & \\
\hline CRP & $1.011(0.999-1.024)$ & 0.075 & & \\
\hline $\mathrm{PNI}<46$ & $2.656(1.256-5.614)$ & 0.011 & $0.328(0.123-0.872)$ & 0.025 \\
\hline
\end{tabular}

SYNTAX: SYNergy between PCI with TAXUS and Cardiac Surgery; SBP: Systolic Blood Pressure; LVEF: Left Ventricular Ejection Fraction; eGFR: Estimated Glomerular Filtration Rate; LDL: Low-density lipoprotein cholesterol; WBC: White blood cell; CRP: C-reactive protein; PNI: Prognostic Nutritional Index

the low-PNI group. The PNI value was a significant independent predictor of all-cause death. Cumulative survival was significantly lower for the patients in the low-PNI group (PNI < 46) relative to the patients in the high-PNI group (PNI $\geq 46$ ). Thus, $\mathrm{PNI}$ on admission appears to be useful for risk stratification of elderly patients with NSTEMI.

It is known that elderly patients have an increased prevalence of poor prognostic factors, such as stroke, diabetes, congestive heart failure and HT
(13). In addition, elderly patients are often malnourished, and malnutrition contributes to poor prognosis by weakening the immune defences of elderly patients in the hospital (14). As a result of the increased risk profile, it may be useful to use different parameters in predicting prognosis.

PNI calculated with the albumin level and the lymphocyte count is used as an indicator of inflammation, immune function and nutritional level (15). Hypoalbuminemia is the result of inflammation, in- 
adequate protein and calorie intake in people with chronic disease. Inflammation and malnutrition reduce the synthesis rate of albumin and increase the deterioration of albumin. Also, the transition of albumin into the extravascular space is increased. As a result, hypoalbuminemia is developed. The albumin level decreases the result of both inflammation and malnutrition in chronic diseases $(16,17)$. Albumin increases the production of prostaglandin-D2 and indirectly causes the inhibition of platelet aggregation (18). Hypoalbuminemia increases blood viscosity and disrupts endothelial functions due to increased free lysophosphatidylcholine levels (19). Albumin is a commonly used measure for nutrition and has been demonstrated to relation with postoperative complications (20). Many reports have demonstrated an elevation of inflammatory markers in myocardial infarction (21) and there is increasing evidence that hypoalbuminemia on admission hospital is a powerful independent predictor of longterm mortality and acute heart failure development in STEMI patients (16).

The effects of malnutrition on the immune system are important. Malnourishment causes lymphatic tissues to develop atrophy, impairs cellular immunity and reduces bactericidal leukocyte activity. Interleukin (IL)-1 activity is suppressed in malnutrition. Impaired IL-1 activity leads to a decrease in the rate of lymphocyte generation (22). Lymphocytes have a crucial effect in regulating the inflammatory response at several stages of the atherosclerotic process (23). In acute coronary events, lymphocytopenia is observed secondary to increased corticosteroid level, and a higher lymphocyte count is considered a more suitable immune reaction and is seen as a stable inflammatory status (24). Lymphocytopenia has been independently associated with major adverse cardiac events and death after myocardial infarction (25). Therefore, a PNI that has been calculated from the serum albumin and lymphocyte counts can predict the nutritional, inflammatory and immune status of elderly patients with NSTEMI.
Long-term mortality rates of NSTEMI have reduced significantly in association with an increase in the frequency of care that includes early interventions (11). There are a few significantly accepted risk scores including TIMI risk score and GRACE score which present precious prognostic information. Age is a parameter for both the GRACE and TIMI risk score (26). Advanced age is accompanied by malnutrition and lower albumin level. A total of 945 elderly patients with STEMI were evaluated during a 2-year follow-up, and malnutrition was detected in approximately $55 \%$ of the participants (27). Therefore, considering that malnutrition increase with age, it can be expressed that data on the evaluation and importance of this in elderly patients with NSTEMI are limited. As presented in recent analyses, lower lymphocyte level is associated with higher mortality (28). PNI combines these two significant measurements and offers valuable prognostic information.

Initially, the PNI was presented to assess the immunological and nutritional aspects of malignity patients (29). It has known that the PNI provides a stronger predictive power of mortality than do its components such as albumin and lymphocyte (30). Then, the PNI score was used as a predictive nutritional marker in patients with different cardiovascular diseases, such as heart failure and STEMI $(7,8)$. Basta et al. demonstrated that in elderly patients with STEMI, a lower PNI level was significantly associated with poor prognosis (27). A recent retrospective observational study of patients with STEMI shown a significant relationship between $\mathrm{PNI}$ and inhospital and long-term mortality $(7,8)$. However, the analysis has been made in elderly STEMI patients who underwent $\mathrm{PCl}$ (8). Therefore, this study has examined the association of PNI with all-cause mortality in elderly patients with NSTEMI.

In this study, we found that PNI was an independent predictor of mortality in elderly patients with NSTEMI. The current study has shown that the low PNI had a higher all-cause mortality rate compared to the high-PNI group, even though both groups 
had similar medical histories, such as DM, heart failure, previous stroke and HPL. A large number of comorbidities are related to nutritional status, and patients in the low PNI group had a higher prevalence of known CAD and previous $\mathrm{HT}$. In this study, patients with low PNI tended to be older, had a higher level of CRP. This partly explains that increased inflammatory activity and decreased serum albumin level may be two of the underlying mechanisms of mortality in elderly patients with NSTEMI. In patients with low PNI, higher CRP levels also confirmed a negative relationship between $\mathrm{PNI}$ and CRP levels in the present study. A previous study has presented that the PNI was independently associated with cardiovascular outcomes after adjusting for traditional risk factors (31). Multivariate Cox hazard models showed that the PNI was independently associated with all-cause mortality after adjusting for risk factors. Therefore, PNI is appropriate to be used in elderly patients with NSTEMI since it is easy and simple to calculate with available practical parameters and less likely to be affected by confounding clinical conditions in coronary artery disease patients such as statin use. Consistent with previous findings $(27,30)$ that low PNI were related to poor prognosis in patients with CAD, the present study more enlarges this association to a particular cohort

\section{REFERENCES}

1. Webster D, Celik O. Systematic review of Kinect applications in elderly care and stroke rehabilitation. J Neuroeng Rehabil. 2014;11:108. (PMID: 24996956).

2. Alexander KP, Roe MT, Chen AY et al. Evolution in cardiovascular care for elderly patients with non-STsegment elevation acute coronary syndromes: results from the CRUSADE National Quality Improvement Initiative. J Am Coll Cardiol. 2005;46(8):1479-87. (PMID: 16226171).

3. Roffi M, Patrono C, Collet JP et al. 2015 ESC Guidelines for the management of acute coronary syndromes in patients presenting without persistent ST-segment elevation: Task Force for the Management of Acute Coronary Syndromes in Patients Pre- such as elderly patients with NSTEMI.

As a result of multivariate Cox regression analysis, it was determined that low PNI and increased heart rate, high WBC, reduced LVEF were associated with the primary endpoint. It has been reported that higher WBC has been used in the estimation of major adverse cardiovascular events in elderly patients (32). In agreement with our study, the study by Kalyoncuoğlu et al. showed that reduced LVEF was an independent predictor of poor prognosis in elderly patients with NSTEMI (33).

This study had several limitations. The present study evaluated the PNI value at admission and did not assess its changes during the follow-up time. This was a retrospective, single-centre, observational study, in which information about the body mass index of patients was not obtained. The range of $\mathrm{PNI}$ values was relatively small. Additional assessments using nutritional indexes may supply beneficial information during the follow-up.

The PNI consists of simple objective measurements that can be easily obtained. Our findings suggest that the PNI score was found to be associated with all-cause mortality in elderly patients with NSTEMI.

senting without Persistent ST-Segment Elevation of the European Society of Cardiology (ESC). Eur Heart J. 2016;37(3):267-315. (PMID: 26320110).

4. Charlton KE, Nichols C, Bowden S et al. Older rehabilitation patients are at high risk of malnutrition: evidence from a large Australian database. J Nutr Health Aging. 2010;14(8):622-8. (PMID: 20922337).

5. Evans C. Malnutrition in the elderly: a multifactorial failure to thrive. Perm J. 2005;9(3):38-41. (PMID: 22811627).

6. Wyszynski DF, Perman M, Crivelli A. Prevalence of hospital malnutrition in Argentina: preliminary results of a population-based study. Nutrition. 2003;19(2):115-9. (PMID: 12591541). 
7. Keskin M, Hayıroğlu MI, Keskin T et al. A novel and useful predictive indicator of prognosis in ST-segment elevation myocardial infarction, the prognostic nutritional index. Nutr Metab Cardiovasc Dis. 2017;27(5):438-46. (PMID: 28392077).

8. Narumi T, Arimoto T, Funayama A et al. Prognostic importance of objective nutritional indexes in patients with chronic heart failure. J Cardiol. 2013;62(5):307-13. (PMID: 23806549).

9. Pathirana AK, Lokunarangoda N, Ranathunga I et al. Prevalence of hospital malnutrition among cardiac patients: results from six nutrition screening tools. Springerplus. 2014;3:412. (PMID: 25143874).

10. Zencirkiran Agus H, Kahraman S. Prognostic nutritional index predicts one-year outcome in heart failure with preserved ejection fraction. Acta Cardiol. 2020;75(5):450-55. (PMID: 31498720).

11. Collet JP, Thiele H, Barbato E et al. 2020 ESC Guidelines for the management of acute coronary syndromes in patients presenting without persistent ST-segment elevation. Eur Heart J. 2020. (PMID: 32860058).

12. Schiller NB, Shah PM, Crawford M et al. Recommendations for quantitation of the left ventricle by two-dimensional echocardiography. American Society of Echocardiography Committee on Standards, Subcommittee on Quantitation of Two-Dimensional Echocardiograms. J Am Soc Echocardiogr. 1989;2(5):358-67. (PMID: 2698218).

13. Gurwitz JH, Osganian V, Goldberg RJ et al. Diagnostic testing in acute myocardial infarction: does patient age influence utilization patterns? The Worcester Heart Attack Study. Am J Epidemiol. 1991;134(9):948-57. (PMID: 1951292).

14. Alzahrani SH, Alamri SH. Prevalence of malnutrition and associated factors among hospitalized elderly patients in King Abdulaziz University Hospital, Jeddah, Saudi Arabia. BMC Geriatr. 2017;17(1):136. (PMID: 28673255).

15. Chan AW, Chan SL, Wong GL et al. Prognostic Nutritional Index (PNI) Predicts Tumor Recurrence of Very Early/Early Stage Hepatocellular Carcinoma After Surgical Resection. Ann Surg Oncol. 2015;22(13):4138-48. (PMID: 25801356).

16. Oduncu $\vee$, Erkol A, Karabay $C Y$ et al. The prognostic value of serum albumin levels on admission in patients with acute ST-segment elevation myocardial infarction undergoing a primary percutaneous coro- nary intervention. Coron Artery Dis. 2013;24(2):88-94. (PMID: 23249632).

17. Ishida S, Hashimoto I, Seike T et al. Serum albumin levels correlate with inflammation rather than nutrition supply in burns patients: a retrospective study. J Med Invest. 2014;61(3-4):361-8. (PMID: 25264055).

18. Gresele P, Deckmyn H, Huybrechts E, Vermylen J. Serum albumin enhances the impairment of platelet aggregation with thromboxane synthase inhibition by increasing the formation of prostaglandin D2. Biochem Pharmacol. 1984;33(13):2083-8. (PMID: 6430299).

19. Joles JA, Willekes-Koolschijn N, Koomans HA. Hypoalbuminemia causes high blood viscosity by increasing red cell lysophosphatidylcholine. Kidney Int. 1997;52(3):761-70. (PMID: 9291198).

20. Relles DM, Richards NG, Bloom JP et al. Serum blood urea nitrogen and serum albumin on the first postoperative day predict pancreatic fistula and major complications after pancreaticoduodenectomy. J Gastrointest Surg. 2013;17(2):326-31. (PMID: 23225108).

21. Danesh J, Collins R, Appleby P, Peto R. Association of fibrinogen, C-reactive protein, albumin, or leukocyte count with coronary heart disease: meta-analyses of prospective studies. Jama. 1998;279(18):147782. (PMID: 9600484).

22. Zhang S, Wan Z, Zhang Y et al. Neutrophil count improves the GRACE risk score prediction of clinical outcomes in patients with ST-elevation myocardial infarction. Atherosclerosis. 2015;241(2):723-8. (PMID: 26132283).

23. Kurtul A, Murat SN, Yarlioglues M et al. Increased neutrophil-to-lymphocyte ratio predicts persistent coronary no-flow after wire insertion in patients with ST-elevation myocardial infarction undergoing primary percutaneous coronary intervention. Clinics (Sao Paulo). 2015;70(1):34-40. (PMID: 25672427).

24. Onsrud M, Thorsby E. Influence of in vivo hydrocortisone on some human blood lymphocyte subpopulations. I. Effect on natural killer cell activity. Scand J Immunol. 1981;13(6):573-9. (PMID: 7313552).

25. Widmer A, Linka AZ, Attenhofer Jost $\mathrm{CH}$ et al. Mechanical complications after myocardial infarction reliably predicted using C-reactive protein levels and lymphocytopenia. Cardiology. 2003;99(1):25-31. (PMID: 12589119).

26. Rossi L, Rosa EM, Guerra MB. GRACE risk score vs 
TIMI risk score. Arq Bras Cardiol. 2011;96(3):257; author reply 57-8. (PMID: 21484071).

27. Basta G, Chatzianagnostou K, Paradossi U et al. The prognostic impact of objective nutritional indices in elderly patients with ST-elevation myocardial infarction undergoing primary coronary intervention. Int $\mathrm{J}$ Cardiol. 2016;221:987-92. (PMID: 27441479).

28. Han YC, Yang TH, Kim DI et al. Neutrophil to Lymphocyte Ratio Predicts Long-Term Clinical Outcomes in Patients with ST-Segment Elevation Myocardial Infarction Undergoing Primary Percutaneous Coronary Intervention. Korean Circ J. 2013;43(2):939. (PMID: 23508782).

29. Yang L, Xia L, Wang $Y$ et al. Low Prognostic Nutritional Index (PNI) Predicts Unfavorable Distant Metastasis-Free Survival in Nasopharyngeal Carcinoma: A Propensity Score-Matched Analysis. PLoS One. 2016;11(7):e0158853. (PMID: 27399281).

30. Chen QJ, Qu HJ, Li DZ et al. Prognostic nutritional index predicts clinical outcome in patients with acute ST-segment elevation myocardial infarction undergoing primary percutaneous coronary intervention. Sci Rep. 2017;7(1):3285. (PMID: 28607442).

31. Wada H, Dohi T, Miyauchi K et al. Prognostic impact of nutritional status assessed by the Controlling $\mathrm{Nu}$ tritional Status score in patients with stable coronary artery disease undergoing percutaneous coronary intervention. Clin Res Cardiol. 2017;106(11):875-83. (PMID: 28634674).

32. Tonet E, Campo G, Maietti E et al. Nutritional status and all-cause mortality in older adults with acute coronary syndrome. Clin Nutr. 2020;39(5):1572-79. (PMID: 31324416).

33. Kalyoncuoğlu M, Katkat F, Biter HI et al. Predicting One-Year Deaths and Major Adverse Vascular Events with the Controlling Nutritional Status Score in Elderly Patients with Non-ST-Elevated Myocardial Infarction Undergoing Percutaneous Coronary Intervention. J Clin Med. 2021;10(11). (PMID: 34067233). 